\title{
Warps and correlations with intrinsic parameters of galaxies in the visible and radio
}

\author{
N. Castro-Rodríguez ${ }^{1,2}$, M. López-Corredoira ${ }^{2}$, M. L. Sánchez-Saavedra ${ }^{3}$, and E. Battaner ${ }^{3}$ \\ 1 Instituto de Astrofísica de Canarias, 38205 La Laguna, Spain \\ 2 Astronomisches Institut der Universität Basel, Venusstrasse 7, Binningen, Switzerland \\ 3 Departamento de Física Teórica y del Cosmos, University of Granada, Avd. Fuentenueva SN., 18002 Granada, Spain
}

Received 15 April 2002 / Accepted 30 May 2002

\begin{abstract}
From a comparison of the different parameters of warped galaxies in the radio, and especially in the visible, we find that:

a) No large galaxy (large mass or radius) has been found to have high amplitude in the warp, and there is no correlation of size/mass with the degree of asymmetry of the warp.

b) The disc density and the ratio of dark to luminous mass show an opposing trend: smaller values give more asymmetric warps in the inner radii (optical warps) but show no correlation with the amplitude of the warp; however, in the external radii is there no correlation with asymmetry.

c) A third anticorrelation appears in a comparison of the amplitude and degree of asymmetry in the warped galaxies.

Hence, it seems that very massive dark matter haloes have nothing to do with the formation of warps but only with the degree of symmetry in the inner radii, and are unrelated to the warp shape for the outermost radii. Denser discs show the same dependence.
\end{abstract}

Key words. galaxies: statistics - galaxies: spiral - galaxies: structure - galaxies: kinematics and dynamics

\section{Introduction}

Many spiral galaxies have warps, disc distortions with an integral-sign shape (S-warp), cup-shape (U-warp), or some form of asymmetry. The Milky Way is an example (Burton 1988, 1992). Indeed, most of the spiral galaxies for which we have relevant information on their structure (because they are edge-on and nearby) show a warp. Sánchez-Saavedra et al. (1990, 2002) and Reshetnikov \& Combes (1998) show that nearly half of the spiral galaxies of selected samples are warped, and many of the rest might also be warped since warps in galaxies with low inclination are difficult to detect. For high redshift, it seems that the effect of warping is even stronger (Reshetnikov et al. 2002).

At present, there are several theories in the literature about the causes of warps in galactic discs. Four remarkable examples of theories which explain the formation of warps are:

- Gravitational tidal effects on a given spiral galaxy due to the presence of a satellite. This does not seem to be enough to induce the observed amplitude in the Galactic warp (Hunter \& Toomre 1969). Weinberg (1998) proposed a mechanism for amplifying the tidal effects caused by a satellite by means of an intermediate massive halo around the galactic disc, but García-Ruiz et al. (2000) have found that the

Send offprint requests to: N. Castro-Rodríguez,

e-mail: ncastro@astro.unibas.ch orientation of the warp is not compatible with the generation of warps by means of this mechanism if the satellites are the Magellanic Clouds, and moreover the magnification of the amplitude is not so high (García Ruiz 2001, Ch. 2). However, this mechanism could operate in galaxies other than the Milky Way.

- The intergalactic magnetic field has been suggested as the cause of galactic warps (Battaner et al. 1990; Battaner et al. 1991; Battaner \& Jiménez-Vicente 1998) directly affecting the gas in the Galactic disc and producing warps in it. Stellar warps in the old population could also be possible as a result of interactions between the gaseous and stellar discs. This gives rise to some interesting predictions, such as the alignment of warps of different galaxies (Battaner et al. 1991) and differences between the stellar and gaseous warps.

- Cosmic infall is invoked to explain the reorientation of a massive Galactic halo, which produces a warp in the disc (Ostriker \& Binney 1989; Jiang \& Binney 1999). This model requires a halo that is much more massive than the disc, an extremely high accretion rate (3 disc masses in 0.9 Gyr; Jiang \& Binney 1999) and, in this scenario, after a sufficiently long time, the angular momentum of the Galaxy to become parallel to the direction of the falling matter causing the warp to decay. It is difficult to understand why the warps are so frequent in this scenario but this difficulty might be overcome by including a prolate halo 
(Ideta et al. 2000), which would prolong the warp's existence. Also, it is difficult to understand how a low-density halo can retain the accreted intergalactic matter. A generic misalignment between halo and the disc (Debattista \& Sellwood 1999) might answer the question, but then we need to think about the reason for the misalignment.

- The accretion of the intergalactic medium directly on to the disc is another possibility (Revaz \& Pfenninger 2001; López-Corredoira et al. 2002). The torque produced in the different rings of the disc by an intergalactic flow of velocity $\sim 100 \mathrm{~km} \mathrm{~s}^{-1}$ and baryon density $\sim 10^{-25} \mathrm{~kg} \mathrm{~m}^{-3}$ is enough to generate the observed warps (López-Corredoira et al. 2002) and also predicts the existence of U-warps (cupshaped), which are less frequent than S- (integral-shaped) warps. Some alignment of the warps in neighbouring galaxies and differences between the gaseous and stellar warps might also be expected. This hypothesis assumes only the existence of a not necessarily homogeneous intergalactic medium with a reasonably low density. Although the idea is plausible and compatible with our observations, there is still no direct proof of the existence of this intergalactic medium.

All these theories make different assumptions about the conditions of the spiral galaxies and their neighbourhood (massive haloes, magnetic fields, intergalactic medium, satellites), so the study of warps becomes interesting as a tool for discriminating among the different scenarios. There are already important works about the observed properties of warps, however we feel that these are inadequate, and that an effort must be made to reduce the number of possible hypotheses. Here, we do not aim to give a final answer to the question but instead present some new correlations that might be useful together with other data to discriminate among the different models.

Some interesting observational results already published are the dependence on the environment (isolated or in clusters) of the warp amplitude asymmetries (differences between the east and west wings of the warp) and frequency (in the visible Reshetnikov \& Combes 1998 and also in the radio GarcíaRuiz 2001; Kuijken \& García-Ruiz 2001). Curiously, more isolated galaxies seem to be more frequently warped (García-Ruiz 2001; Kuijken \& García-Ruiz 2001) and this would be against the gravitational interaction of satellites, at least in some cases. Halo-disk misalignments without external dependence are also excluded. It seems that intergalactic magnetic fields, or the accretion of intergalactic matter on to either the halo or the disc are better representations. Therefore, there already exists in the literature some papers which have correlated the warp characterictics with the environmental parameters. We are not going to explore these correlations again, but rather focus on the correlations with the intrinsic parameters of galaxies. For instance, one interesting question now is whether there is any correlation between halo properties and warp amplitude/asymmetry.

If the halo were an important element in the formation of warps, we should observe some dependence on it. Some models have a warp amplitude depending on the halo mass. Apart from the hypotheses which talk about a halo as an intermediary between external forces and the disc (Weinberg 1998;
Jiang \& Binney 1999), works by Nelson \& Tremaine (1995) or Debattista \& Sellwood (1999) predict that, although in most cases the dynamical friction between the disc and the halo damps the warp, it can also excite the warp. This is the reason why we will try to analyze the optical and radio warps in the present paper through the correlations with the mass/luminosity ratios derived from the rotation curves (provided they are related to the fraction of dark matter in the galaxies). We also produce correlations with other parameters that represent the intrinsic size of the galaxy (radius, mass, or luminosity).

\section{Data}

This study is based on two samples of galaxies, one of 228 galaxies in optical bands (see Table 1) and the other of 26 galaxies in radio (see Table 2). We have completed the information on the warp amplitudes with some intrinsic parameters of the galaxies.

\subsection{Optical data}

The optical warp measurements come from Sánchez-Saavedra et al. (1990, 2002), who have analysed images obtained from the Palomar Observatory Sky Survey (POSS) and the DSS. They measured the amplitude of the warp in galaxies mostly from the southern hemisphere (Sánchez-Saavedra et al. 1990, 2002); however, we also took some data from the northern hemisphere (Sánchez-Saavedra et al. 1990). The galaxies were selected according to the following criteria:

- high surface brightness $(B<14.5)$;

- galaxies large enough to detect the warp. The galaxies have a $\log R_{25} \geq 24 \operatorname{arcsec}\left(R_{25}\right.$ is the radius of the angular size of the isophote $\left.\mu=25 \mathrm{mag} / \operatorname{arcsec}^{2}\right)$;

- morphological type, $T$, between 0 and 7;

- inclination angle greater than 75 degrees.

We took these data and sought several intrinsic parameters of each galaxy in the literature. The $H$-band $(\lambda 1.6 \mu \mathrm{m})$ magnitude was used because it is a good mass tracer of the stellar population. Moreover, the luminous mass in NIR bands is not much affected by dust and gas extinction as the visible bands are and is less contaminated by the young population of the spiral arms. The total luminosity of a galaxy near $2 \mu \mathrm{m}$ is thought to be a better tracer of the stellar mass than the visible (which is biased by recent star formation) or the far-infrared (biased again by recent star formation, which creates and heats dust grains that emit thermally in this waveband; Jablonka \& Arimoto 1992). We then tried to find some correlations between these intrinsic parameters and the warp amplitudes in our sample. In Table 1 are shown all the galaxies with these parameters. The columns list the following information:

- Column 1: PGC and NGC number.

- Columns 2 and 3: Warp amplitudes extracted from Sánchez-Saavedra et al. (1990, 2002). The first number is the amplitude on the east side of the galaxy and the second 
Table 1. Optical data. Columns in the table represent: name of the galaxy, warp amplitude in the west and east side of the galaxy, redshift, maximum rotation velocity, $\log \left(D_{25}\right), H$ magnitude and distance.

\begin{tabular}{|c|c|c|c|c|c|c|c|}
\hline PGC/NGC & East WA & West WA & $\begin{array}{l}c z \\
\left(\mathrm{~km} \mathrm{~s}^{-1}\right)\end{array}$ & $\begin{array}{l}V_{\text {rot }} \\
\left(\mathrm{km} \mathrm{s}^{-1}\right)\end{array}$ & $\begin{array}{l}\log D_{25} \\
0.1 \operatorname{arcmin}\end{array}$ & $m_{\mathrm{H}}$ & $\begin{array}{l}d \\
(\mathrm{Mpc}) \\
\end{array}$ \\
\hline PGC 474 & $\overline{13}$ & 12 & $\overline{\left.1542_{i}\right)}$ & $139_{i)}$ & 1.53 & $\overline{-}$ & 20.6 \\
\hline PGC 627 & 13 & 13 & 1495 a) & $77_{1)}$ & 1.39 & - & $20.0^{*}$ \\
\hline PGC 725 & 19 & 17 & $6004_{a)}$ & $226_{1)}$ & 1.34 & - & 80.0 \\
\hline PGC 1851 & 20 & 14 & $1596_{a)}$ & $233_{1)}$ & 1.92 & 7.6 & 21.3 \\
\hline PGC 1942 & 8 & 13 & $7110_{h)}$ & $108_{h)}$ & 1.41 & 10.1 & 94.8 \\
\hline PGC 1952 & 15 & 12 & $2626_{a)}$ & $205_{1)}$ & 1.45 & - & 35.0 \\
\hline PGC 2228 & 13 & 12 & $3043 h)$ & $115_{1)}$ & 1.31 & 11.6 & 40.6 \\
\hline PGC 2482 & 15 & 17 & $3946_{h)}$ & $291_{h)}$ & 1.45 & 9.2 & 52.6 \\
\hline PGC 2789 & 12 & 15 & $241_{g)}$ & $204_{4)}$ & 2.43 & 4.5 & $3.4^{*}$ \\
\hline PGC 2800 & 13 & 13 & $5765_{h)}$ & $219_{h)}$ & 1.24 & - & 76.9 \\
\hline PGC 2805 & 25 & 17 & $1345_{c)}$ & $59{ }_{s)}$ & 1.48 & - & 17.9 \\
\hline PGC 3743 & 14 & 12 & $2290 a)$ & $172_{a)}$ & 1.57 & - & 30.5 \\
\hline PGC 4440 & 13 & 20 & 3552 a) & $198_{1)}$ & 1.41 & 9.8 & 47.4 \\
\hline PGC 4912 & 17 & 25 & $5883 a)$ & $234_{1)}$ & 1.29 & 10.1 & 40.6 \\
\hline PGC 5688 & 9 & 5 & $5431_{h)}$ & $255_{h)}$ & 1.34 & 9.8 & 72.4 \\
\hline PGC 6966 & 4 & 12 & $5005_{h)}$ & $257_{1)}$ & 1.48 & - & 66.7 \\
\hline PGC 7306 & 13 & 13 & $4443 h)$ & $136_{h)}$ & 1.29 & - & 59.2 \\
\hline PGC 7427 & 6 & 13 & $5530 a)$ & $178_{1)}$ & 1.27 & - & 73.7 \\
\hline PGC 8326 & 7 & 8 & $8133 a)$ & $312_{1)}$ & 1.40 & 10.2 & 108.4 \\
\hline PGC 8673 & 9 & 10 & $1890_{h)}$ & $96_{1)}$ & 1.34 & - & 25.2 \\
\hline PGC 9582 & 5 & 11 & $4773_{h)}$ & $294_{1)}$ & 1.33 & - & 63.6 \\
\hline PGC 10965 & 7 & 7 & $2065_{a)}$ & $160_{1)}$ & 1.57 & - & 27.5 \\
\hline PGC 11198 & 25 & 20 & $4495_{h)}$ & $211_{h)}$ & 1.45 & - & 59.9 \\
\hline PGC 11595 & 18 & 18 & $1391_{a)}$ & $83_{1)}^{n}$ & 1.47 & 12.0 & 18.5 \\
\hline PGC 11659 & 10 & 10 & $5529 a)$ & $255_{1)}$ & 1.36 & 9.8 & 73.7 \\
\hline PGC 11851 & 9 & 6 & $1318_{a)}$ & $116_{1)}$ & 1.53 & 10.2 & 17.5 \\
\hline PGC 12521 & 15 & 10 & 3949 a) & $178_{1)}$ & 1.34 & 10.5 & 52.6 \\
\hline PGC 13171 & 14 & 10 & $1812_{h)}$ & $109 h)$ & 1.40 & 10.1 & 24.1 \\
\hline PGC 13458 & 9 & 6 & 1068 a) & $150_{1)}$ & 1.59 & 9.2 & 14.2 \\
\hline PGC 13569 & 0 & 0 & $1638 a)$ & $65_{1)}$ & 1.36 & 11.2 & 21.8 \\
\hline PGC 13646 & 12 & 8 & $2168_{C)}$ & $168_{1)}$ & 1.50 & - & 28.9 \\
\hline PGC 13727 & 15 & 14 & $1179 a)$ & 193 1) & 1.88 & 8.3 & 15.7 \\
\hline PGC 13809 & 6 & 0 & $1882 a)$ & $131_{1)}$ & 1.69 & 9.4 & 25.0 \\
\hline PGC 13912 & 9 & 9 & $980_{a)}$ & $120_{1)}$ & 1.63 & - & - \\
\hline PGC 14071 & 9 & 9 & $1050_{a)}$ & $84_{h)}$ & 1.40 & - & 14.0 \\
\hline PGC 14190 & 7 & 16 & 1279 a) & 95 1) & 1.56 & - & 17.0 \\
\hline PGC 14255 & 13 & 13 & $1291_{a)}$ & $95_{1)}$ & 1.28 & 11.3 & 17.2 \\
\hline PGC 14259 & 9 & 13 & $4111_{a)}$ & $175_{1)}$ & 1.43 & 10.8 & 54.8 \\
\hline PGC 14337 & 0 & 0 & $5386_{a)}$ & $182_{1)}$ & 1.31 & 10.7 & 71.8 \\
\hline PGC 14337 & 0 & 0 & $5386_{a)}$ & $182_{1)}$ & 1.31 & 10.7 & 72.0 \\
\hline PGC 14397 & 8 & 8 & $1094_{a)}$ & $190_{1)}$ & 1.72 & - & 14.6 \\
\hline PGC 14824 & 7 & 10 & $1359_{C)}$ & $92_{1)}$ & 1.59 & - & 18.1 \\
\hline PGC 15455 & 9 & 8 & $1851_{a)}$ & $120_{1)}$ & 1.44 & - & 24.6 \\
\hline PGC 15635 & 9 & 17 & $4852 h)$ & $288_{h)}$ & 1.52 & - & 64.6 \\
\hline PGC 15654 & 10 & 10 & $4759 h)$ & $225_{h)}$ & 1.37 & - & 63.4 \\
\hline PGC 15674 & 14 & 15 & $3705_{h)}$ & $202_{h)}$ & 1.27 & 10.0 & 49.4 \\
\hline PGC 15749 & 20 & 20 & $1678_{a)}$ & $92_{1)}$ & 1.38 & - & 22.4 \\
\hline PGC 16144 & 0 & 0 & $2819 h)$ & $155_{h)}$ & 1.35 & 10.7 & 37.6 \\
\hline
\end{tabular}

number is the warp amplitude on the west side. These values divided by 100 give the tangent of the angle. This is the angle between the galactic centre and the end of the warp.

- Column 4: Redshift of each galaxy taken from the NED. Each reference is specified in the table.
- Column 5: Rotation velocity of the galaxy at $R_{25}$, which is more or less the maximum rotation velocity. This parameter comes from several sources, mainly from Mathewson et al. (1992) and Persic \& Salucci (1995). Each reference is specified in the columns of the table. 
Table 1. continued.

\begin{tabular}{|c|c|c|c|c|c|c|c|}
\hline$\overline{\mathrm{PGC} / \mathrm{NGC}}$ & East WA & West WA & $\begin{array}{l}c z \\
\left(\mathrm{~km} \mathrm{~s}^{-1}\right)\end{array}$ & $\begin{array}{l}V_{\text {rot }} \\
\left(\mathrm{km} \mathrm{s}^{-1}\right)\end{array}$ & $\begin{array}{l}\log D_{25} \\
0.1 \text { arcmin }\end{array}$ & $m_{\mathrm{H}}$ & $\begin{array}{l}d \\
(\mathrm{Mpc})\end{array}$ \\
\hline$\overline{\text { PGC } 16168}$ & 7 & 7 & 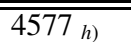 & 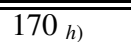 & "1.22 & $\overline{-1}$ & $\bar{~} 61.0$ \\
\hline PGC 16199 & 12 & 12 & $1169_{a)}$ & $87_{1)}$ & 1.44 & - & 15.6 \\
\hline PGC 16636 & 0 & 0 & $4329 h)$ & $199 h)$ & 1.49 & - & 57.7 \\
\hline PGC 17056 & 10 & 10 & $2828 a_{a)}$ & $172_{1)}$ & 1.24 & - & 37.7 \\
\hline PGC 17174 & 0 & 0 & $1755_{a)}$ & $158_{h)}$ & 1.51 & - & 23.4 \\
\hline PGC 17969 & 10 & 10 & $2382_{h)}$ & $145_{1)}$ & 1.34 & 10.5 & 31.8 \\
\hline PGC 18437 & 6 & 5 & $1228 a)$ & $137_{1)}$ & 1.60 & 9.6 & 16.4 \\
\hline PGC 18765 & 0 & 0 & $1696_{i)}^{a)}$ & $143_{a)}$ & 1.52 & 9.8 & 22.6 \\
\hline PGC 19996 & 5 & 5 & $2681_{h)}$ & $166_{f)}^{a)}$ & 1.35 & 10.1 & 35.7 \\
\hline PGC 21815 & 6 & 6 & $1131_{b)}$ & $98_{b)}$ & 1.67 & 8.4 & 15.1 \\
\hline PGC 21822 & 17 & 7 & $3237_{h)}$ & $245_{h)}$ & 1.48 & 9.3 & 43.1 \\
\hline PGC 22272 & 0 & 0 & $1558_{h)}$ & $130 h)$ & 1.45 & 11.1 & 20.8 \\
\hline PGC 22338 & 8 & 9 & 1119 ii) & $148_{m)}$ & 1.80 & - & $14.9^{*}$ \\
\hline PGC 22910 & 13 & 12 & $5954_{a)}$ & $223_{1)}$ & 1.41 & - & 79.4 \\
\hline PGC 23558 & 20 & 20 & $1776_{h)}$ & $91_{h)}$ & 1.27 & - & 23.7 \\
\hline PGC 23992 & 15 & 17 & $4533 a)$ & $190_{1)}$ & 1.15 & - & 60.4 \\
\hline PGC 24685 & 6 & 8 & $4570_{a)}$ & $308_{1)}$ & 1.48 & 9.4 & 60.9 \\
\hline PGC 25886 & 9 & 9 & $1838_{h)}$ & $256_{h)}$ & 1.63 & - & 24.5 \\
\hline PGC 25926 & 3 & 3 & $2178_{a)}$ & $158_{a)}$ & 1.65 & - & 29.0 \\
\hline PGC 26561 & 10 & 10 & $1640 a)$ & $245_{1)}$ & 1.75 & - & 21.8 \\
\hline PGC 27135 & 0 & 0 & $929_{i)}$ & $100_{1)}$ & 1.86 & - & $6.40^{*}$ \\
\hline PGC 27735 & 13 & 13 & $4449_{a)}$ & $171_{1)}$ & 1.28 & - & 59.3 \\
\hline PGC 28117 & 17 & 27 & $4315_{a)}$ & $170_{1)}$ & 1.41 & - & 57.5 \\
\hline PGC 28246 & 17 & 20 & $2893 a)$ & $183_{1)}$ & 1.50 & - & 38.5 \\
\hline PGC 28283 & 0 & 0 & $2868 h)$ & $220_{h)}$ & 1.59 & - & 38.2 \\
\hline PGC 28778 & 8 & 8 & $2697 a)$ & $154_{i)}$ & 1.40 & - & 36.0 \\
\hline PGC 28840 & 7 & 8 & $2802_{a)}$ & $123_{1)}$ & 1.53 & - & 37.3 \\
\hline PGC 28909 & 0 & 0 & $2520_{a)}$ & $208_{1)}$ & 1.83 & - & 33.6 \\
\hline PGC 29691 & 0 & 0 & $2840_{h)}$ & $142_{1)}$ & 1.35 & - & 37.9 \\
\hline PGC 29716 & 0 & 0 & $2526_{v)}$ & $161_{k)}$ & 1.59 & - & 33.7 \\
\hline PGC 29743 & 9 & 9 & $2603 j)$ & $164_{1)}$ & 1.50 & - & 34.7 \\
\hline PGC 29841 & 0 & 0 & $3603 h)$ & $185_{1)}$ & 1.32 & 10.5 & 48.0 \\
\hline PGC 30716 & 0 & 0 & $3138_{a)}$ & $160_{1)}$ & 1.30 & - & 41.8 \\
\hline PGC 31154 & 0 & 0 & $3608_{a)}^{a)}$ & $254_{1)}$ & 1.35 & - & 48.1 \\
\hline PGC 31426 & 10 & 6 & $5042_{C)}$ & $290 u$ & 1.41 & - & 67.2 \\
\hline PGC 31677 & 10 & 0 & $3756_{h)}$ & $204_{1)}$ & 1.50 & - & 50.1 \\
\hline PGC 31723 & 11 & 0 & $4152_{h)}$ & 169 1) & 1.32 & - & 55.4 \\
\hline PGC 31919 & 0 & 0 & $1032 a)$ & $60_{1)}$ & 1.46 & 11.8 & 13.8 \\
\hline PGC 31995 & 8 & 8 & 2932 a) & $185_{1)}$ & 1.45 & - & 39.1 \\
\hline PGC 32271 & 6 & 7 & $3047 h)$ & $218_{h)}$ & 1.54 & - & 40.6 \\
\hline PGC 32328 & 0 & 0 & $5704_{a)}$ & $245_{1)}$ & 1.30 & - & 76.0 \\
\hline PGC 32550 & 6 & 0 & $\left.3108_{j}\right)$ & $114_{a)}$ & 1.54 & - & 41.4 \\
\hline PGC 35861 & 25 & 25 & $2702_{h)}^{\prime \prime}$ & $241_{h)}$ & 1.53 & - & 36.0 \\
\hline PGC 36315 & 10 & 6 & $3701_{a)}$ & $136_{1)}$ & 1.21 & - & 49.3 \\
\hline PGC 37178 & 0 & 0 & $2013 a)$ & $141_{1)}$ & 1.60 & 9.7 & 26.8 \\
\hline PGC 37243 & 5 & 4 & $2944 a)$ & $176_{1)}$ & 1.42 & - & 39.2 \\
\hline PGC 37271 & 7 & 6 & $1702 a)$ & $123 a)$ & 1.64 & - & 22.7 \\
\hline PGC 37304 & 9 & 9 & 5715 a) & $254_{1)}$ & 1.36 & - & 76.2 \\
\hline PGC 37334 & 0 & 0 & 2889 b) & $162_{b)}$ & 1.42 & - & 38.5 \\
\hline PGC 38426 & 12 & 11 & $4476_{C)}$ & $198_{1)}$ & 1.31 & - & 59.7 \\
\hline PGC 38464 & 5 & 9 & 1728 a) & $121_{1)}$ & 1.38 & - & 23.0 \\
\hline PGC 38841 & 0 & 0 & 3133 a) & $150_{h)}$ & 1.31 & - & 41.8 \\
\hline PGC 40023 & 0 & 0 & $2940 a)$ & $\left.237_{j}\right)$ & 1.50 & - & 39.2 \\
\hline PGC 40284 & 17 & 19 & $2002_{a)}$ & $176_{1)}$ & 1.49 & 9.3 & 26.7 \\
\hline PGC 42684 & 0 & 0 & $5502_{a)}$ & $221_{1)}$ & 1.32 & - & 73.4 \\
\hline PGC 42747 & 13 & 20 & 3210 a) & $145_{1)}$ & 1.42 & 11.2 & 42.8 \\
\hline PGC 43021 & 0 & 0 & $5260 a)$ & $278_{1)}$ & 1.41 & 10.0 & 70.1 \\
\hline PGC 43224 & 10 & 10 & $3211_{h)}$ & $162_{h)}$ & 1.25 & 10.3 & 42.8 \\
\hline PGC 43313 & 0 & 0 & $3693_{C)}$ & $209 u)$ & 1.40 & 10. & 49.2 \\
\hline PGC 43330 & 14 & 16 & $1408_{c)}$ & $60_{2)}$ & 1.47 & 10.3 & 18.8 \\
\hline
\end{tabular}


Table 1. continued.

\begin{tabular}{|c|c|c|c|c|c|c|c|}
\hline$\overline{\mathrm{PGC} / \mathrm{NGC}}$ & East WA & West WA & $\begin{array}{l}c z \\
\left(\mathrm{~km} \mathrm{~s}^{-1}\right)\end{array}$ & $\begin{array}{l}V_{\text {rot }} \\
\left(\mathrm{km} \mathrm{s}^{-1}\right)\end{array}$ & $\begin{array}{l}\log D_{25} \\
0.1 \text { arcmin }\end{array}$ & $m_{\mathrm{H}}$ & $\begin{array}{l}d \\
(\mathrm{Mpc})\end{array}$ \\
\hline PGC 43342 & 0 & 0 & $4459_{h)}$ & $254_{h)}$ & 1.39 & - & 59.4 \\
\hline PGC 43679 & 0 & 0 & $2258_{i}$ & $\left.106_{j}\right)$ & 1.39 & - & 30.1 \\
\hline PGC 44254 & 0 & 0 & $2839_{c)}$ & $142 u)$ & 1.28 & 10.7 & 37.8 \\
\hline PGC 44271 & 0 & 0 & $3376_{a)}$ & $172_{1)}$ & 1.43 & - & 45.0 \\
\hline PGC 44358 & 0 & 0 & $1487_{c)}$ & $114_{i)}$ & 1.51 & - & 19.8 \\
\hline PGC 44409 & 0 & 0 & $2173_{a)}$ & 184 & 1.67 & - & 29.0 \\
\hline PGC 44931 & 8 & 7 & $3812_{c)}$ & $2011)$ & 1.45 & - & 50.8 \\
\hline PGC 44966 & 0 & 0 & 4995 a) & $231_{1)}$ & 1.19 & - & 66.6 \\
\hline PGC 45006 & 9 & 13 & $4527_{c)}$ & $206_{1)}$ & 1.42 & - & 60.4 \\
\hline PGC 45098 & 12 & 9 & $2896_{a)}$ & $169_{1)}$ & 1.46 & - & 38.6 \\
\hline PGC 45127 & 10 & 10 & $4007_{h)}$ & $180_{1)}$ & 1.27 & 10.7 & 53.4 \\
\hline PGC 45279 & 14 & 14 & $560 a)$ & $180_{a)}$ & 2.31 & 7.5 & $6.7^{*}$ \\
\hline PGC 45487 & 0 & 0 & $2621_{a)}$ & $114_{1)}$ & 1.48 & - & 34.9 \\
\hline PGC 45911 & 0 & 0 & $2754_{a)}$ & $143_{1)}$ & 1.47 & - & 36.7 \\
\hline PGC 45952 & 0 & 0 & $3006_{a)}$ & $170_{1)}$ & 1.38 & - & 40.1 \\
\hline PGC 46441 & 10 & 10 & $2744_{d)}$ & $191_{2)}$ & 1.54 & - & 36.6 \\
\hline PGC 46650 & 4 & 15 & $2566_{e)}$ & $131_{a)}$ & 1.46 & - & 34.2 \\
\hline PGC 46768 & 0 & 0 & $2256_{a)}$ & 112 & 1.25 & - & 30.1 \\
\hline PGC 47345 & 7 & 14 & $3604_{h)}$ & $207_{h)}$ & 1.52 & - & 48.0 \\
\hline PGC 47394 & 0 & 0 & $1503_{a)}$ & $251_{a)}$ & 1.91 & 8.6 & 20.0 \\
\hline PGC 47948 & 8 & 9 & $2577 a)$ & $158_{1)}$ & 1.40 & - & 34.4 \\
\hline PGC 48359 & 0 & 0 & $3631_{v)}$ & $229_{1)}$ & 1.31 & - & 48.4 \\
\hline PGC 49129 & 9 & 15 & $141_{a)}$ & $47_{a)}$ & 1.41 & - & - \\
\hline PGC 49190 & 17 & 15 & $3931_{h)}$ & $93_{h)}$ & 1.23 & - & 52.4 \\
\hline PGC 49586 & 8 & 8 & $2760_{a)}$ & $196_{b)}$ & 1.45 & - & 36.8 \\
\hline PGC 49676 & 11 & 13 & $2663 a)$ & $241_{a)}$ & 1.76 & 8.7 & 35.5 \\
\hline PGC 49836 & 4 & 10 & $2907 a)$ & $153_{1)}$ & 1.37 & - & 38.8 \\
\hline PGC 50676 & 14 & 15 & $1541_{a)}$ & $112_{1)}$ & 1.64 & 10.9 & 20.5 \\
\hline PGC 50798 & 0 & 0 & $3017_{a)}$ & $164_{1)}$ & 1.41 & - & 40.2 \\
\hline PGC 51613 & 0 & 0 & $2245_{a)}$ & $123_{1)}$ & 1.48 & - & 29.9 \\
\hline PGC 52410 & 0 & 0 & $2869 a)$ & $174_{1)}$ & 1.35 & - & 38.2 \\
\hline PGC 52411 & 9 & 9 & 3420 a) & $215_{1)}$ & 1.45 & 9.5 & 45.6 \\
\hline PGC 52991 & 0 & 0 & $2945 a)$ & $99_{1)}$ & 1.30 & 11.3 & 39.3 \\
\hline PGC 53361 & 0 & 0 & $4510 a)$ & $152_{1)}$ & 1.36 & - & 60.1 \\
\hline PGC 54392 & 0 & 0 & $522_{m)}$ & 79 5) & 2.05 & - & $7.0^{*}$ \\
\hline PGC 54637 & 9 & 12 & $4655_{a)}$ & $212_{1)}$ & 1.40 & - & 62.0 \\
\hline PGC 56077 & 0 & 0 & $2692_{a)}$ & $115_{1)}$ & 1.30 & - & 35.9 \\
\hline PGC 57582 & 0 & 0 & $\left.2044_{f}\right)$ & $169_{i)}$ & 1.78 & - & 27.2 \\
\hline PGC 57876 & 0 & 0 & $3410_{a)}$ & $2221)$ & 1.59 & - & 45.5 \\
\hline PGC 59635 & 0 & 0 & $1508_{a)}$ & $101_{1)}$ & 1.57 & - & 20.1 \\
\hline PGC 60216 & 15 & 15 & 2859 a) & $109_{1)}$ & 1.30 & - & 38.1 \\
\hline PGC 60595 & 0 & 0 & $4698_{a)}$ & $190_{1)}$ & 1.39 & - & 62.6 \\
\hline PGC 62024 & 0 & 0 & $3183_{a)}$ & $2011)$ & 1.22 & - & 42.4 \\
\hline PGC 62706 & 0 & 0 & $3182_{a)}$ & $133_{1)}$ & 1.57 & - & 42.4 \\
\hline PGC 62782 & 8 & 4 & $1841_{a)}$ & $83_{1)}$ & 1.47 & - & 24.5 \\
\hline PGC 62816 & 10 & 10 & $5024_{a)}$ & $233_{1)}$ & 1.25 & - & 66.9 \\
\hline PGC 62922 & 7 & 0 & $4404_{a)}$ & $280_{1)}$ & 1.57 & - & 58.7 \\
\hline PGC 62964 & 13 & 13 & $2847 a)$ & $241_{4)}$ & 1.62 & - & 38.0 \\
\hline PGC 63395 & 3 & 6 & $1928_{a)}$ & 117 a) & 1.51 & - & 25.7 \\
\hline PGC 63577 & 12 & 12 & $4231_{a)}$ & $138_{1)}$ & 1.29 & - & 56.4 \\
\hline PGC 64597 & 5 & 5 & $4196_{a)}$ & $120_{a)}$ & 1.34 & - & 55.9 \\
\hline PGC 65794 & 9 & 3 & $9150_{a)}$ & $323_{1)}$ & 1.42 & - & 122.0 \\
\hline PGC 65915 & 11 & 8 & $3122 a)$ & $177_{1)}$ & 1.53 & - & 41.6 \\
\hline PGC 66530 & 14 & 7 & $3144_{a)}$ & $266_{1)}$ & 1.50 & - & 41.9 \\
\hline PGC 66617 & 0 & 0 & $2715_{r}$ & $101_{1)}$ & 1.25 & - & 36.2 \\
\hline PGC 66836 & 0 & 0 & $797 d)$ & $73_{1)}$ & 1.52 & - & $16.2^{*}$ \\
\hline PGC 67045 & 0 & 0 & $857 d)$ & $96_{3)}$ & 1.89 & 9.3 & $16.0^{*}$ \\
\hline PGC 67078 & 0 & 0 & $2479_{a)}$ & $85_{1)}$ & 1.30 & - & 33.0 \\
\hline PGC 67158 & 0 & 7 & $3400 a)$ & $175_{1)}$ & 1.44 & 10.3 & 45.3 \\
\hline PGC 67904 & 6 & 5 & 2635 a) & $264_{1)}$ & 1.78 & 8.5 & 35.1 \\
\hline
\end{tabular}


Table 1. continued.

\begin{tabular}{|c|c|c|c|c|c|c|c|}
\hline PGC/NGC & East WA & West WA & $\begin{array}{l}c z \\
\left(\mathrm{~km} \mathrm{~s}^{-1}\right)\end{array}$ & $\begin{array}{l}V_{\text {rot }} \\
\left(\mathrm{km} \mathrm{s}^{-1}\right)\end{array}$ & $\begin{array}{l}\log D_{25} \\
0.1 \text { arcmin }\end{array}$ & $m_{\mathrm{H}}$ & $\begin{array}{l}d \\
(\mathrm{Mpc})\end{array}$ \\
\hline PGC 68223 & 11 & 11 & $2847_{r)}$ & $169_{r)}$ & 1.38 & 10.1 & 38.0 \\
\hline PGC 68389 & 6 & 5 & $1746_{a)}$ & $174_{1)}$ & 1.64 & - & 23.3 \\
\hline PGC 69161 & 19 & 18 & $\left.2091_{k}\right)$ & $117_{1)}$ & 1.55 & 10.9 & 27.9 \\
\hline PGC 69539 & 9 & 8 & $1240_{a)}$ & $102_{1)}$ & 1.60 & 10.7 & 16.5 \\
\hline PGC 69661 & 16 & 11 & $2360_{a)}$ & $175_{1)}$ & 1.48 & 9.8 & 31.5 \\
\hline PGC 69707 & 0 & 0 & $2364_{a)}$ & $100_{1)}$ & 1.59 & - & 31.5 \\
\hline PGC 69967 & 0 & 0 & $3001_{a)}$ & $148_{1)}$ & 1.41 & 10.5 & 40.0 \\
\hline PGC 70025 & 7 & 7 & $2857 n)$ & $\left.167_{f}\right)$ & 1.50 & - & 38.1 \\
\hline PGC 70070 & 0 & 0 & $1681_{a)}$ & $109_{1)}$ & 1.58 & - & 22.4 \\
\hline PGC 70081 & 9 & 9 & $1940 a)$ & $240_{1)}$ & 1.49 & - & 25.9 \\
\hline PGC 70084 & 13 & 7 & $5041_{a)}$ & $308_{a)}$ & 1.31 & - & 67.2 \\
\hline PGC 70324 & 0 & 0 & $1059_{a)}$ & $85_{a)}$ & 1.62 & 10.1 & 14.1 \\
\hline PGC 71800 & 7 & 0 & $2008_{a)}$ & $101_{a)}$ & 1.24 & - & 26.7 \\
\hline PGC 71948 & 0 & 0 & $2876_{a)}$ & $253_{1)}$ & 1.74 & - & 38.3 \\
\hline PGC 72178 & 4 & 8 & $1489 a)$ & $110_{1)}$ & 1.43 & - & 19.8 \\
\hline NGC 4013 & 5 & 5 & $834_{f)}$ & $193_{1)}$ & 1.72 & 8.7 & $12.0^{*}$ \\
\hline NGC 1560 & 5 & 5 & $-36_{d)}$ & $76_{1)}$ & 1.99 & 9.4 & $3.0^{*}$ \\
\hline NGC 2654 & 8 & 8 & $1347_{f)}$ & $197_{1)}$ & 1.63 & - & 22.4 \\
\hline NGC 2683 & 7 & 7 & $411_{f)}$ & $275_{1)}$ & 1.97 & 6.8 & $5.1^{*}$ \\
\hline NGC 2820 & 12 & 16 & $3811_{o}$ & $210_{1)}$ & 1.46 & 9.5 & 50.8 \\
\hline NGC 2820 & 12 & 16 & $\left.3811_{o}\right)$ & $210_{1)}$ & 1.46 & 9.5 & 50.8 \\
\hline NGC 3510 & 10 & 10 & $705_{l l)}$ & $83_{1)}$ & 1.58 & 11.2 & $9.0^{*}$ \\
\hline NGC 3628 & 16 & 16 & $843_{s)}$ & $223_{1)}$ & 2.17 & 6.9 & $6.7^{*}$ \\
\hline NGC 4010 & 6 & 6 & $907_{i)}$ & $118_{1)}$ & 1.62 & 10.2 & $11.0^{*}$ \\
\hline NGC 4565 & 2 & 2 & $1282_{t)}$ & 259 1) & 2.21 & 6.7 & 10.0 \\
\hline NGC 6045 & 11 & 11 & $9986_{a)}$ & $258_{1)}$ & 1.12 & 10.9 & 133.1 \\
\hline NGC 6161 & 14 & 12 & $5904_{a)}$ & $256_{1)}$ & 1.29 & 10.2 & 78.7 \\
\hline NGC 6242 & 13 & 13 & $4620_{a)}$ & $172_{1)}$ & 1.28 & - & 61.6 \\
\hline NGC 7640 & 7 & 7 & $369_{f)}$ & $110_{1)}$ & 2.03 & 9.3 & $9.2^{*}$ \\
\hline
\end{tabular}

The references of each value are: * Huchtmeier et al. (1989); ${ }^{a)}$ Mathewson et al. (1996); ${ }^{b}$ Di Nella et al. (1996); ${ }^{c)}$ Da Costa et al. (1998); ${ }^{d)}$ Saunders et al. (2000); ${ }^{e)}$ Longmore et al. (1982); ${ }^{f)}$ Haynes et al. (1998); ${ }^{g)}$ Huchtmeier et al. (1985); ${ }^{h)}$ Theureau et al. (1998); ${ }^{i)}$ Fisher et al. (1981); ${ }^{i i)}$ Tully (1988); ${ }^{j}$ Richter et al. (1987); $\left.{ }^{k}\right)$ Davies et al. (1989); ${ }^{l)}$ de Vaucouleurs et al. (1991), ${ }^{l l)}$ Thuan et al. (1981); ${ }^{m)}$ Strauss et al. (1992); ${ }^{n)}$ Fairall et al. (1988); ${ }^{o)}$ Staveley-Smith et al. (1987); ${ }^{p)}$ Dressler et al. (1991); ${ }^{q)}$ Fairall et al. (1991); ${ }^{r)}$ Chengalur et al. (1993); ${ }^{s)}$ Tifft et al. (1988); ${ }^{t)}$ Giovanelli et al. (1997); ${ }^{u}$ Fairall et al. (1992); ${ }^{v)}$ Bottinelli et al. (1993); ${ }^{w)}$ Loveday et al. (1996); ${ }^{1)}$ Mathewson et al. (1992); ${ }^{2)}$ Staveley-Smith et al. (1988); ${ }^{3)}$ Reif et al. (1982); ${ }^{4)}$ Corradi et al. (1991); ${ }^{5)}$ Banks et al. (1999).

- Column 6: The values of $\log D_{25}$ extracted from the LEDA database, where $D_{25}$ is the diameter of the isophote with $25 \mathrm{mag} / \operatorname{arcsec}^{2}$ in units of 0.1 arcmin.

- Column 7: Magnitude in $H$ from 2MASS (Jarret et al. 2000 and de Vaucouleurs \& Longo 1988).

- Column 8: Distance to the galaxy, obtained using the redshift value and the Tully-Fisher relation with a Hubble constant of $75 \mathrm{~km} \mathrm{~s}^{-1} \mathrm{Mpc}^{-1}$. This method is not very accurate for very close galaxies; therefore, when the redshift is less than $1000 \mathrm{~km} \mathrm{~s}^{-1}$, we used distances from Huchtmeier \& Richter (1989).

With this information, we sought any correlation between the amplitude of the warp or the difference between the east side and west side, and mass/luminosity, dimensions, infrared luminosity or total mass of the galaxies derived from the rotation curves. The results are commented on in Sect. 3.

\subsection{Radio data}

Radio data are from García-Ruiz (2001). There are only 26 galaxies with measurements of warps at these wavelengths. All the information is given in Table 2; the meaning of each column is the same as for the optical data. There are no other important works on radio warp amplitudes in the literature. In most cases, the warp is more prominent in radio observations than in optical images because the former extends to greater galactocentric distances. The galaxies were selected according the following criteria:

- listed in the Upsala General Catalogue of Galaxies (Nilson 1973);

- galaxies from the norther hemisphere with declinations higher than 20 degrees;

- blue diameters greater than $1.5^{\prime}$;

- optic inclination angles greater than 75 degrees;

- flux density higher than $100 \mathrm{mJy}$ in the radio. 

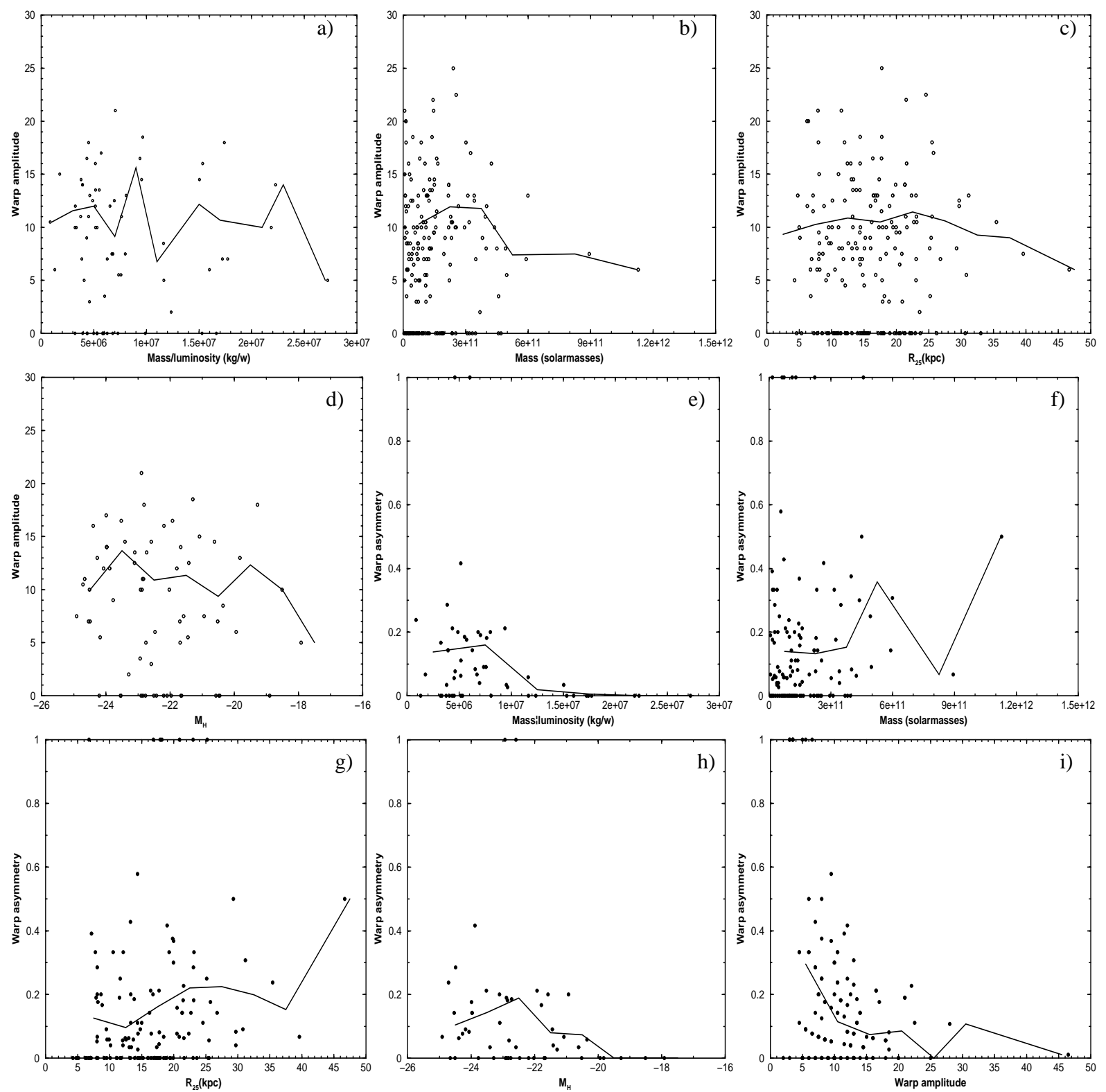

Fig. 1. Optical data from Sánchez-Saavedra et al. (2002). The panels represent from top to bottom: mass/luminosity versus warp amplitude, total mass in solar masses versus warp amplitude, $R_{25}$ versus warp amplitude, absolute magnitude versus warp amplitude, Mass/luminosity versus warp asymmetry, total mass in solar masses versus warp asymmetry, $R_{25}$ versus warp asymmetry for optical sample, absolute magnitude versus warp asymmetry and amplitude versus warp asymmetry. The points represent each galaxy in the sample and the line is the average of the galaxies with amplitude $>3$, taking a given width of the bin in the $x$ axis.

Again, we have performed the same analysis as in the previous section for optical data, as described in the following section.

\section{Analysis of the correlations}

With the information available in the tables we can determine $R_{25}$ (kpc) from the angular size and the distance, and the mass $M=R_{25} v^{2} / G$. The luminosity (or absolute magnitude) is also immediately derived once we know the apparent magnitude and the distance. We define the amplitude as the $\frac{1}{2}$ (East WA + West WA) and the asymmetry as
|East WA - West WA|/(East WA + West WA). In this section, we analyse the correlations among the different quantities.

Our results are represented in Figs. 1 and 2 for optical and radio warps respectively. For each one, we have two different sets of plots, graphs of warp amplitudes and graphs of the warp asymmetries against intrinsic parameters of the galaxies. The following parameters are represented:

- warp amplitude against the mass-luminosity relation. The total mass in kilograms that was calculated with the maximum of the rotational velocity curve, the radius of the isophote with $25 \mathrm{mag} / \operatorname{arcsec}^{2}$ in $\mathrm{kpc}$ and the absolute magnitude in $H$ for the reasons given in Sect. 2; 

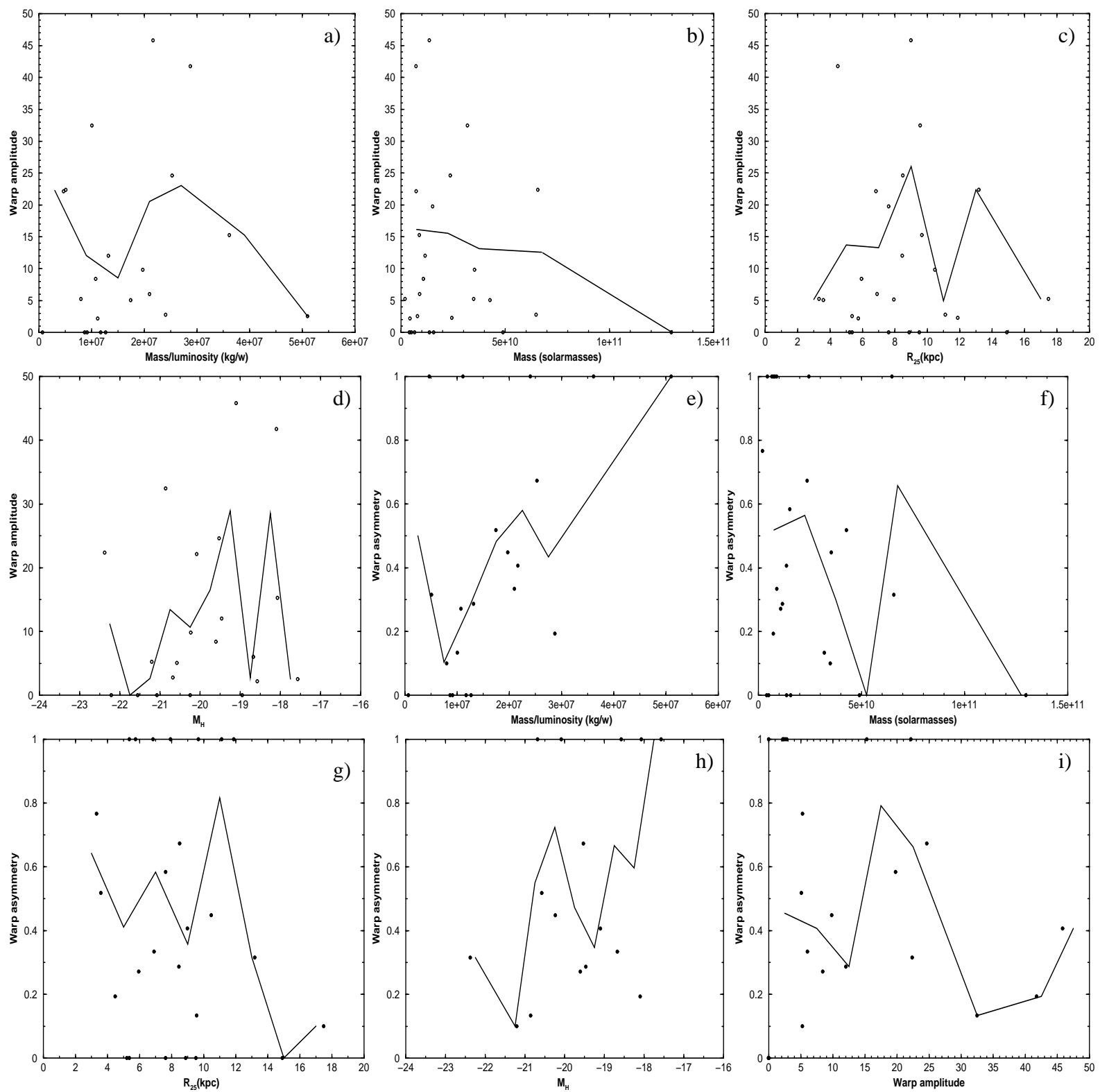

Fig. 2. Radio data from García-Ruiz (2001). The panels represent from top to bottom: mass/luminosity versus warp amplitude, total mass in solar masses versus warp amplitude, $R_{25}$ versus warp amplitude, absolute magnitude versus warp amplitude, mass/luminosity versus warp asymmetry, total mass in solar masses versus warp asymmetry, $R_{25}$ versus warp asymmetry for optical sample, absolute magnitude versus warp asymmetry and amplitude versus warp asymmetry. The points represent each galaxy in the sample and the line is the average of the galaxies, taking a given width of the bin in the $x$ axis.

- warp asymmetry against the same quantities;

- the relation between the warp amplitude and the asymmetry of the warps comparing their east and west wings.

In Figs. 1 and 2 are represented the relations between the parameters of the warp (amplitude and asymmetry) against: mass-luminosity relation (Figs. 1a and 1e for its relation with the warp amplitude and the warp asymmetry respectively; and $2 \mathrm{a}$ and $2 \mathrm{e}$ ), the total mass (Figs. $1 \mathrm{~b}$ and $1 \mathrm{f}$; and $2 \mathrm{~b}$ and $2 \mathrm{f}$ ), $R_{25}$ (Figs. 1c and $1 \mathrm{~g}$; and $2 \mathrm{c}$ and g2), the absolute magnitude in $H$ (Figs. $1 \mathrm{~d}$ and $1 \mathrm{~h}$; and $2 \mathrm{~d}$ and $\mathrm{h} 2$ ). Finally, Figs. $1 \mathrm{i}$ and $2 \mathrm{i}$ represent the relation between the warp amplitude and the warp asymmetry. The points represent each galaxy of the sample of Sánchez-Saavedra et al. (1990, 2002) and García-Ruiz (2001). The number of points in each plot depends on the number of available galaxies with information on the two variables represented. The solid line represents the average of the warp amplitude and warp asymmetry respectively along the $x$ axis and was determined with data for the warp amplitude between 3 and 30 for optical data. We try to avoid galaxies with a small warp that might introduce errors in the measurements. In the case of the radio data all the measurements are presented in the average representation. Here, we have calculated the average value (solid line) with warp amplitudes between 0 and 50 for two reasons: there are fewer galaxies at this wavelength and 
we cannot discard any of them; it is easier to measure the warp in the radio than in the optical bands. In the case of the warp asymmetry graphs, the average value is between 0 and 1 . In all cases, ten points along the $x$ axis have been used to fit this value. The total number of galaxies is 228 for the optical data and 26 for the radio data, but sometimes there is no parameter (in $H$ band because 2MASS is not yet complete, the rotational velocity, etc.) for all galaxies (see Tables 1 and 2). In these cases, the figures show a lower number of galaxies. The data displayed in the figures show the following behaviour:

- In the relation between mean warp angle and massluminosity relation, we have not found any correlation in either the visible or the radio. Points are distributed in all ranges of $M / L$. Large oscillations in the average value (solid line) reinforce this conclusion. In Fig. 2a there is a fall but is due to only one point with $M / L \sim 5 \times 10^{7}$.

- However, in the representations of the amplitude versus total mass, $R_{25}$ (both parameters are related since larger galaxies are more massive) there is a slight anticorrelation, more pronounced in the visible and more conspicuous in the plot of amplitude vs. mass. Higher values of mass and $R_{25}$ have on average a smaller warp angle. There is an absence of galaxies in all the figures for highmass/radius galaxies and high-amplitude warps. For instance, we can see that all the galaxies with mass greater than $4-5 \times 10^{11} M_{\odot}$ tend to have smaller warps. This anticorrelation is clearer in Fig. 1b, where the solid line falls for large masses. This behaviour is not so clear in Fig. 1c because we have a large concentration of points for small $x$ values and tends to smooth the fall of the solid line. There are small values of $x$ and a low number of galaxies in the radio data (Figs. 2b, 2c), so no correlation can be seen in this region. In general, galaxies from the García-Ruiz (2001) sample are nearer and smaller.

- In asymmetry representations, we see almost the opposite behaviour. There are no apparent correlations with mass or radius (see Figs. 1f and $1 \mathrm{~g}$ ). There are many oscillations in the average value for Figs. 1f, $1 \mathrm{~g}, 2 \mathrm{f}$ and $2 \mathrm{~g}$, and there is no clear tendency for either the mass or the radio to grow in either sample. But there is a clear anticorrelation in the massluminosity relation and in the absolute magnitude representation for visible warps. For Figs. 1e and $1 \mathrm{~h}$ there is a slight drop around $10^{7} \mathrm{~kg} \mathrm{~W}^{-1}$ and $-21 \mathrm{mag}$, respectively. For larger $M / L$ ratios, the galaxies have less asymmetry in their warps (see Fig. 1e). For low $M / L$ values, the galaxies have a high dispersion in asymmetry of between 0 and 0.4 . The solid line represents this behaviour. In Figs. $2 \mathrm{e}$ and $2 \mathrm{~h}$ we cannot see this anticorrelation, or, if there is one, it would be opposite to the trend in the visible warps. If we consider the last points with warp asymmetry equal to 1 and a large $M / L$ ratio (see Fig. $2 \mathrm{e}$ ), the solid line tends to rise. There are not many points in this region and the total sample is very poor. The optical data are more complete. The same behaviour is seen in Fig. $2 \mathrm{~h}$.

- In Figs. 1i and 2i, the asymmetry of the warp amplitude in the east and west side against warp amplitude has been represented. For larger warp amplitudes we have less asymmetry. An analogous result is shown in García-Ruiz (2001), who used only radio data and found that warp amplitudes are more prominent than in optical images. We must bear in mind that the errors in the measurements are proportional to $\sqrt{2} S_{\mathrm{a}} / A$, where $S_{\mathrm{a}}$ is the error in the measurements and $A$ is the average value. This means that for lower amplitudes the error will be larger, and this could introduce scatter in the results. In any case, the average value of the asymmetry should not be affected by this scatter, so we can tentatively talk about the detection of an anticorrelation between both variables.

All these relations are subject to the authenticity of the warp characteristics measured by Sánchez-Saavedra et al. (1990, 2002) and García-Ruiz (2001), especially for the visible warps, since these are more likely to be confused with other features (spiral arms, for instance). Nonetheless, the possible contamination, if reasonably small (no more than $20 \%$ of the sample), would only introduce some noise in the correlations. Unless most of the data are wrong, it cannot be expected that the present features are caused by this contamination.

\section{Discussion and conclusions}

In our analysis of the correlations between warp characteristics and other parameters of the galaxies we find some trends of correlation or anticorrelation in some cases and nothing in other cases. The number of galaxies is not very large, so possible minor systematic errors in the parameters are not totally discarded, and the dispersion of values is large, so the correlations among the different parameters is not perfect (we have no correlation factor close to 1). In any case, we think that these relations reveal some real characteristics which can be tentatively examined as follows:

- There is a slight anticorrelation between the amplitude of the warp in both directions (average between the east and west amplitudes) and parameters such as the total mass, luminosity and radius. We believe that this is to be expected for any mechanism that produces a warp as a reaction to an external torque, whatever it its origin (gravitational torque, magnetic torque, accretion torque; see López-Corredoira et al. 2002). More massive (i.e. generally larger and more luminous) galaxies have a more massive disc which forces the warped rings of the disc to collapse towards the flat disc. The more massive the disc is, the larger are the internal countertorques of the disc and the smaller the amplitude (López-Corredoira et al. 2002).

- There is no correlation between the amplitude of the warp and the mass-luminosity relation. This negative result is indeed very informative. If the halo were the predominant effect in the dynamics responsible of the formation of the warp, we would expect a larger amplitude for higher mass/luminosity ratios (a larger fraction of dark matter embedded in the halo). Either the rotation curve velocity is not related to the total mass of the galaxy or the warp amplitude is independent of the relative proportion of halo mass. Bosma (1991) found that galaxies with small dark halo core 
Table 2. Radio data. Columns in the table represent: name of the galaxy, warp amplitude in the west and east side of the galaxy, redshift, maximum rotation velocity, $\log \left(D_{25}\right), H$ magnitude and distance.

\begin{tabular}{llllllll}
\hline \hline UGC & EAST WA & WEST WA & $\begin{array}{l}c z \\
\mathrm{~km} / \mathrm{sg}\end{array}$ & $\begin{array}{l}V_{\text {rot }} \\
\mathrm{km} / \mathrm{sg}\end{array}$ & $\begin{array}{l}\log D_{25} \\
0.1 \mathrm{arcmin}\end{array}$ & $m_{\mathrm{H}}$ & $\begin{array}{l}d \\
\mathrm{Mpc}\end{array}$ \\
\hline \hline 1281 & 1.22 & 9.27 & 157 & 50 & 1.65 & - & 5.1 \\
2549 & 2.44 & 7.69 & 10355 & 226 & .83 & 12.2 & 36.3 \\
3137 & 5.76 & 4.71 & 992 & 93 & 1.55 & $* 11.4$ & 33.8 \\
3909 & 15.48 & 8.57 & 945 & 77 & 1.37 & 12.5 & 24.5 \\
4278 & 5.06 & 0.00 & 560 & 79 & 1.66 & 11.9 & 8.1 \\
4806 & 0.00 & 5.59 & 1947 & 158 & 1.56 & $* 10.9$ & 21.1 \\
5452 & 31.33 & 8.22 & 1342 & 93 & 1.38 & - & 21.7 \\
5459 & 5.41 & 14.23 & 1112 & 120 & 1.66 & $* 10.8$ & 15.9 \\
5986 & 41.21 & 8.04 & 615 & 109 & 1.84 & 10.1 & 8.5 \\
6126 & 49.85 & 33.65 & 704 & 83 & 1.54 & 11.6 & 8.8 \\
6283 & 6.11 & 10.68 & 719 & 88 & 1.56 & 10.7 & 11.3 \\
6964 & 28.10 & 36.79 & 905 & 120 & 1.59 & 10.3 & 16.9 \\
7089 & 0.00 & 0.00 & 774 & 57 & 1.50 & 8.8 & 11.6 \\
7090 & 0.00 & 0.00 & 560 & 149 & 1.81 & $* 8.9$ & 10.2 \\
7125 & 10.33 & 0.00 & 1071 & 59 & 1.64 & - & 12.6 \\
7151 & 0.00 & 0.00 & 267 & 64 & 1.78 & 9.9 & 6.0 \\
7321 & 4.54 & 0.00 & 409 & 94 & 1.74 & - & 14.9 \\
7483 & 0.00 & 0.00 & 1248 & 94 & 1.47 & 10.9 & 17.6 \\
7774 & 64.44 & 27.16 & 526 & 80 & 1.48 & 12.5 & 20.6 \\
8246 & 30.57 & 0.00 & 794 & 63 & 1.53 & 13.4 & 19.4 \\
8286 & 8.04 & 4.01 & 407 & 75 & 1.77 & $* 10.8$ & 8.0 \\
8396 & 44.31 & 0.00 & 945 & 68 & 1.23 & 12.1 & 27.5 \\
8550 & 0.00 & 4.36 & 364 & 57 & 1.48 & 12.0 & 13.2 \\
8709 & 0.00 & 0.00 & 2402 & 194 & 1.71 & 9.3 & 19.8 \\
8711 & 15.30 & 29.43 & 1531 & 146 & 1.60 & 9.4 & 22.5 \\
9242 & 0.00 & 0.00 & 1436 & 81 & 1.68 & - & 12.6 \\
\hline & & & & & & & \\
\hline
\end{tabular}

The references of each value are: Cols. 1-3 from García-Ruiz (2001), the warp amplitudes are in the same units than optical amplitudes; Cols. 4-6 from García-Ruiz (2001) and LEDA database; Col. 7 from 2MASS and galaxies with (*) Tormen et al. (1995); Col. 8 from GarcíaRuiz (2001).

radii (as determined from rotation curve decomposition) are less likely to be warped, but this could be due to an indirect dependence on the scales. The fact here is that larger fraction of dark mass in the galaxy do not relate to the amplitude of the warps.

- There is no correlation between the asymmetry of the warp (differences between the east and west amplitudes) and the total mass and radius. This means that the reasons for the asymmetry are mainly external (satellites, accretion, intergalactic magnetic fields) and are independent of the size of the galaxy.

- There is an anticorrelation between the asymmetry of the warp and the mass-luminosity relation and perhaps also with the luminosity of the stellar population in the disc, but only for optical warps in both cases. In the radio there is no clear correlation, or, if there is one, it is opposite to the behaviour in the visible. This result is somewhat puzzling. It seems to indicate that the halo is responsible in some degree for the symmetry of the warp in the inner part, which is visible in the optical. The luminosity density or mass density in the disc would also be related with the degree of asymmetry (since there is no correlation with the radius and there is an anticorrelation with the total luminosity, it seems that the luminosity density is the factor to be related with the symmetry). The more massive the halo is with respect the rest of the galaxy and the more luminous the disc is (within a constant radius), the more symmetric it is in its inner parts. However, in its outer parts, visible in the radio, the symmetry seems to be independent of these factors. It seems that the forces which produce the asymmetry in the warp (interaction with other satellites, combination of U- and S-warps, etc.) are predominant in the most external radii with respect the halo forces, which tend to produce the symmetry; that is, the asymmetry due to external forces is effected at larger radii for smaller dark mass fractions. As a matter of fact, we have a clear example of this behaviour in our own Galaxy: the gas warp observed in the radio (Burton 1988 ) is clearly symmetric for $R<1.6 R_{\odot}$ but asymmetric for $R>1.6 R_{\odot}\left(R_{\odot} \approx 8 \mathrm{kpc}\right.$; López-Corredoira et al. 2000). In our Galaxy, this radius of transition is equal to $13 \mathrm{kpc}$, which is precisely the value of $R_{25}$ (Goodwin et al. 1998). A tentative explanation for this would be that the halo mass distribution, reflected in the rotation curves, plays a major role for $R<R_{25}$; outer rotation curves are not caused by the presence of a massive halo but have another explanation (magnetic fields, MOND, etc.; see Battaner \& Florido 2000). In any case, if a very massive halo existed well beyond $R_{25}$ it is clear that the asymmetry could not be reduced 
as for $R<R_{25}$ so its dynamical effects must be negligible with respect to the forces that produce the asymmetries.

- Galaxies with larger amplitudes are more symmetric. This is another observational fact that must be accounted for by any theory which tries to explain asymmetric warps. If we interpret the asymmetries as a superposition of S- and Uwarps, we regard the relative contribution of the U-warp as lower for larger absolute values of S-warps. For instance, in the theory of the accretion of intergalactic matter on to the disc (López-Corredoira et al. 2002) this would mean that large warps were produced only when the direction of the infalling flow is far from the galactic pole, and this provides also small asymmetries. If the asymmetries were produced by the presence of a companion galaxy, that would mean that the typical gravitational forces are comparable to the forces that produce S-warps with small amplitudes, and that they become unimportant for large S-warps.

Summing up, we think that the correlations analysed here can give us some clues about the predominant mechanism for the formation of warps in spiral galaxies. At present, the data seem to indicate that the role of the halo is important only in making $\mathrm{S}$-warps at $R<R_{25}$ more symmetric, and that asymmetries are more important in less warped galaxies. This favours scenarios in which the halo is not very important in the formation of S-warps, especially radio S-warps and is in agreement with theories that identify the origin of the warps as directly related to external (intergalactic) factors without the mediation of the halo. The origin of the asymmetries in the warps might be different from the mechanism of S-warps, and in such a case the halo could play a role, only in the inner region.

In the introduction, we have described four different theories to explain the formation of warps. The present results cannot give a definitive answer about which is the correct one. Our goal in the present paper is just to present observational results, not to defend or deny a particular theory. As a consequence of these results, a few words can be added to the comparisons between theories and observations:

- Gravitational interaction with a satellite: this can be the mechanism, but only if there are nearby satellites massive enough to produce the observed warps without any amplification of the halo as an intermediary, which seems not to be the case in many warped galaxies (for instance, Milky Way and many apparently isolated galaxies).

- Intergalactic magnetic field: this is in general consistent with the present results. A slight anticorrelation between asymmetry and mass/luminous relation is seen in Fig. 1e in $R<R_{25}$. Under this hypothesis, the asymmetry could be due to inhomogeneous intergalactic fields or to other perturbative effects, even of a non-magnetic nature. If the asymmetries are really driven by a magnetic field mechanism, the anticorrelation found would suggest that this mechanism would also have an influence on rotation curves.

- Misalignment of the halo: this should produce some correlations of the warp amplitude with the mass-luminosity ratio, which are not observed. Hence, unless an explanation can be found for this non-correlation, it seems that this theory should be discarded.
- Direct accretion of intergalactic medium onto the disc: this explains the present results, but need either to have a dark matter halo within $R<R_{25}$ which has some effect on the amplitude of U-warps, or flat rotation curves are produced by the same matter accretion onto the disc which is responsible of the U-warps.

These are just attempted interpretations in the light of the present results. It is also possible that several mechanisms can be present in the warp formation at the same time. With further data for more galaxies, at higher resolution, and with a more detailed theoretical analysis of the different hypotheses to fit the observations, these results and interpretations can be corroborated and/or improved. New work with optical, infrared and radio data could be very useful for confirming the present trend and to reduce the dispersion of values.

Acknowledgements. Thanks are given to Victor P. Debattista and A. Guijarro. This article makes use of data products from 2MASS, which is a joint project of the Univ. of Massachusetts and the Infrared Processing and Analysis Center, funded by the NASA and the NSF. This work has been supported by "Cajacanarias" (Canary Islands, Spain) and the project AYA2000-2046-Co2-02 of the spanish MCYT.

\section{References}

Banks, G. D., Disney, M. J., Knezek, P. M., et al. 1999, ApJ, 524, 612 Battaner, E., Florido, E., \& Sanchez-Saavedra, M. L. 1990, A\&A, 236, 1

Battaner, E., Garrido, J. L., Sanchez-Saavedra, M. L., \& Florido, E. 1991, A\&A, 251, 402

Battaner, E., \& Jimenez-Vicente, J. 1998, A\&A, 332, 809

Battaner, E., \& Florido, E. 2000, Fund. Cosmic Phys., 21, 1

Bosma, A. 1991, Warped Disks and Inclined Rings around Galaxies, ed. S. Casertano, P. D. Sackett, \& F. Briggs (Cambridge Univ. Press, Cambridge), 181

Bottinelli, L., Durand, N., Fouque, P., et al. 1993, A\&AS, 102, 57

Burton, W. B. 1988, Galactic and Extragalactic Radio Astronomy, ed. K. I. Kellerman, \& G. L. Verschuur (Springer-Verlag, Berlin), 295

Burton, W. B. 1992, The Galactic Interstellar Medium, ed. D. Pfenniger, \& P. Bartholdi (Springer-Verlag, Berlin), 126

Chengalur, J. N., Salpeter, E. E., \& Terzian, Y. 1993, ApJ, 419, 30

Corradi, R. L. M., \& Capaccioli, M. 1991, A\&AS, 90,

Da Costa, L. N., Willmer, C. N. A., Pellegrini, et al. 1998, AJ, 116, 1

Davies, R. D., Staveley-Smith, L., \& Murray, J. D. 1989, MNRAS, 236,171

Debattista, V., \& Sellwood, J. 1999, ApJ, 513, L107

de Vaucouleurs, G., \& Longo, G. 1988, Catalogue of visual and infrared photometry of galaxies from $0.5 \mu \mathrm{m}$ to $10 \mu \mathrm{m}$ (1961-1985) (The University of Texas, Texas)

de Vaucouleurs, G. 1991, RC3-Third Reference Catalogue of Bright Galaxies (Springer-Verlag)

Di Nella, H., Paturel, G., Walsh, A. J., Bottinelli, L., Gouguenheim, L., \& Theureau, G. 1996, A\&AS, 118, 311

Dressler, A. 1991, ApJS, 75, 241

Fairall, A. P. 1988, MNRAS, 230, 69

Fairall, A., \& Jones, A. 1991, Southern Redshifts Catalogue, Publications of the Department of Astronomy, University of Cape Town, num. 11 (Radio)

Fairall, A., Willmer, C. N. A., Calderon, J. H., et al. 1992, AJ, 103, 11

Fisher, J. R., \& Tully, R. B. 1981, ApJS, 47, 139 
García-Ruiz, I., Kuijken, K., \& Dubinski, J. 2000, MNRAS, submitted, preprint [astro-ph/0002057]

García-Ruiz, J. 2001, Ph.D. Thesis, University of Groningen, The Netherlands

Giovanelli, R., Avera, E., \& Karachentsev, I. D. 1997, AJ, 114, 122

Goodwin, S. P., Gribbin, J. \& Hendry, M. A. 1998, Obs., 118, 201

Haynes, M. P., Hogg, D. E., Maddalena, R. J., Roberts, M. S., \& Van Zee, L. 1998, AJ, 115, 62

Huchtmeier, W. K., \& Seiradakis, J. H. 1985, A\&A, 143, 216

Huchtmeier, W. K., \& Richter, O. G. 1989, A general catalog of HI observations of galaxies (Springer-Verlag, New York)

Hunter, C., \& Toomre, A. 1969, ApJ, 155, 747

Ideta, M., Hozumi, S., Tsuchiya, T., \& Takizawa, M. 2000, MNRAS, 311,733

Jablonka, P., \& Arimoto, N. 1992, A\&A, 255, 63

Jarret, T. H., Chester, T., Cutri, R., et al. 2000, AJ, 119, 2498

Jiang, I., \& Binney, J. 1999, MNRAS, 303, L7

Kuijken, K., \& García-Ruiz, I. 2001, Galaxy Disks and Disk Galaxies, ed. J. G. Funes, S. J., \& E. Maria Corsini (Astronomical Society of the Pacific, San Francisco), 230, 401

Longmore, A. J., Hawarden, T. G., Mebold, U., \& Webster, B. L. 1982, MNRAS, 200, 325

López-Corredoira, M., Hammersley, P. L., Garzón, F., Simonneau, E., \& Mahoney, T. J. 2000, MNRAS, 313, 392

López-Corredoira, M., Betancort-Rijo, J., \& Beckman, J. E. 2002, A\&A, 386, 169

Loveday, J., Peterson, B. A., Maddox, S. J., \& Efstathiou, G. 1996, ApJS, 107, 201

Mathewson, D. S., Ford, V. L., \& Buchhorn, M. 1992, ApJS, 81, 413

Mathewson, D. S., \& Ford, V. L. 1996, ApJS, 107, 97

Nelson, R. W., \& Tremaine, S. 1995, MNRAS, 275, 897
Nilson, P. 1973, Uppsala general catalogue of galaxies, Astronomiska Observatorium, Uppsala

Ostriker, J., \& Binney, J. 1989, MNRAS, 237, 785

Persic, M., \& Salucci, P. 1995, ApJS, 99, 501

Reif, K., Mebold, U., Goss, W. M., van Woerden, H., \& Siegman, B. 1982, A\&AS, 50, 451

Reshetnikov, V., \& Combes, F. 1998, A\&A, 337, 9

Reshetnikov, V., Battaner, E., Combes, F., \& Jiménez-Vicente, J. 2002, A\&A, 382, 513

Revaz, Y., \& Pfenninger, D. 2001, Gas and Galaxy Evolution, ed. J. E. Hibbard, M. Rupen, \& J. H. van Gorkom (San Francisco: Astronomical Society of the Pacific), ASP Conf. Proc., 240 , 278

Richter, O. G., \& Huchtmeier, W. K. 1987, A\&AS, 68, 427

Sánchez-Saavedra, M. L., Battaner, E., \& Florido, E. 1990, MNRAS, 246,458

Sánchez-Saavedra, M. L., Battaner, E., Guijarro, A., López-Corredoira, M., \& Castro-Rodríguez, N. 2002, A\&A, submitted

Saunders, W., Sutherland, W. J., \& Maddox, S. J. 2000, MNRAS, 317, 55

Staveley-Smith, L., \& Davies, R. D. 1987, MNRAS, 224, 953

Staveley-Smith, L., \& Davies, R. D. 1988, MNRAS, 231, 833

Strauss, M. A., Huchra, J. P., Davis, M., et al. 1992, ApJS, 83, 29

Theureau, G., Bottinelli, L., Coudreau-Durand, N., et al. 1998, A\&AS, 130,333

Thuan, T. X., \& Martin, G. E. 1981, ApJ, 247, 823

Tifft, W. G., \& Cocke, W. J. 1988, ApJS, 67, 1

Tormen, G., \& Burstein, D. 1995, ApJS, 96, 123

Tully, R. B. 1988, Nearby Galaxies Catalog (Cambridge University Press)

Weinberg, M. 1998, MNRAS, 299, 499 